Quantitative MRI in patients with idiopathic generalized epilepsy demonstrated subtle, but widespread, cerebral structural changes (focal cerebral dysgenesis) not identified on routine MRI in a study at the Institute of Neurology, London, UK (Woermann FG, Sisodiya SM, Free SL, Duncan JS. Brain Sept 1998;121:1661-7).

\title{
FOCAL CORTICAL OPIOIDS AND READING EPILEPSY
}

The release of endogenous opioids in 5 patients with reading-induced seizures was investigated using C-diprenorphine PET scans at the MRC Cyclotron Unit Hammersmith Hospital, and the Institute of Neurology, London, UK. During reading a scientific paper, opioid-receptor binding in the left parieto-temporooccipital cortex (Brodmann area 37) was increased in control patients and decreased in those with reading epilepsy. Opioid-like substances may be involved in the termination of reading-induced seizures. (Koepp MJ, Richardson MP, Brooks DJ, Duncan JS. Focal cortical release of endogenous opioids during readinginduced seizures. Lancet Sept 19;352:952-55). (Respond: Prof JS Duncan, National Society for Epilepsy and Institute of Neurology, 33 Queen Square, London WC1N 3BG, UK).

COMMENT. This novel PET approach to the measurement of neurotransmitter changes associated with focal seizure activity during readinginduced seizures provides further information regarding the anatomical localization of a specific learning disability.

\section{SPET SCAN ABNORMALITIES IN EPILEPTIC APHASIA}

SPET scans, using Tc-exametezime, EEG and MRI were evaluated in 25 children with language deficits associated with epilepsy treated at the Royal Hospital for Sick Children, Edinburgh, UK. Seizures, with onset between 0.3 and 12 years (mean, $4 \mathrm{yrs}$ ), included atypical absence in 15 and tonic-clonic in 10. All had epileptiform EEGs, with enhanced abnormalities in sleep in 16. MRI was abnormal in 6 , including tuberous sclerosis cortical lesions in 1 , stroke in 1 , cortical dysplasia (1), temporal sclerosis (3). SPET scans were abnormal and hypometabolic in 22, bilateral in 7 , and anterior, mainly frontal and temporal, but variable in localization in 15 . Aphasia was receptive in 24 , expressive in 20 , and nominal in 8 . The acquired communication disorder, with onset between 1.5 and 12 years (mean, 6 yrs), did not meet strict criteria for Landau-Kleffner syndrome. Clinical and/or EEG seizure activity were responsive to clobazam or nitrazepam in 11 patients, and ACTH, alone or with benzodiazepine, was effective in 19 . Benzodiazepine sensitivity testing employed in 21 under EEG control was positive in 18 and negative in 3. An encephalopathy secondary to a persistent epileptic discharge and characterized by regional hypometabolism on SPET scan was thought to underly the onset of acquired aphasia. (O'Regan ME, Brown JK, Goodwin GM, Clarke M. Epileptic aphasia: a consequence of regional hypometabolic encephalopathy? Dev Med Child Neurol 1998;40:508-516). (Respond: Dr ME O'Regan, Department of Paediatric Neurology, Royal Hospital for Sick Children, 9 Sciennes Rd, Edinburgh, EH9 1LF, UK).

COMMENT. Acquired epileptic aphasia in young children may be induced by the epileptic focus, as suggested by Deonna (1991). The Edinburgh SPET- and EEGmonitored study supports this hypothesis, finding evidence for a regional hypometabolic encephalopathy secondary to a persistent epileptic discharge, and advocating treatment and suppression of the EEG epileptiform activity, with or without concomitant clinical seizures. In epileptic aphasia we attempt to treat the 
EEG as well as any associated clinical seizures. Unfortunately, my success rate in a small number treated in Chicago cannot compare with that reported in the large series from Edinburgh. Benzodiazepines, the "fortnight drugs" (Dr Cynthia Stack's apt title), frequently lose effectiveness after 2 weeks, and ACTH has a high relapse rate.

Language and motor reorganization following early left hemisphere lesions was investigated using oxygen 15-water PET scans in 9 patients (aged 4-20 years) studied at Wayne State University Medical School, Detroit, MI ( Muller R-A, Rothermel RD, Chugani HT et al. Arch Neurol Aug 1998;55:1113-9). All patients had epilepsy and received antiepileptic medication. Regional cerebral blood flow was studied during rest, listening to sentences, repetition of sentences, and finger tapping of the right hand. Differential reorganizational patterns following early left hemisphere lesions found a greater potential for homotopic interhemispheric reorganization in language than in motor domains.

\section{CONCOMITANT ABSENCE AND ROLANDIC EPILEPSY}

Of 80 children with absence epilepsy (CAE) and 42 with Rolandic epilepsy (BECCT), five ( 3 girls and 2 boys) had both syndromes, in a study at the University of Berne, Switzerland. All 5 patients had both generalized synchronous $3 / \mathrm{sec}$ spike and wave complexes and centrotemporal spikes in the EEGs, but the EEG finding did not always correspond with the clinical presentation. Only 2 patients showed both EEG patterns and both types of clinical seizures, absence and focal motor. Clinical manifestation of absences and partial motor seizures in the same patient is extremely rare. (Ramelli GP, Donati F, Moser H, Vassella F. Concomitance of childhood absence and rolandic epilepsy. Clin Electroencephalogr Oct 1998;29:177-180). (Reprints: Gian Paolo Ramelli MD, Division of Child Neurology, Department of Pediatrics, Inselspital, University of Berne, CH-3010 Berne, Switzerland).

COMMENT. My colleague, Dr Cynthia Stack (Director of Neurophysiology and Electroencephalography at Children's Memorial Hospital), is in agreement with the rarity of concomitant occurrence of these two common forms of epilepsy. She would prefer to see simultaneous EEG and video recordings to confirm the concomitant clinical manifestations of the syndromes, and questions whether the use of carbamazepine to treat the partial seizures might explain precipitation of a concurrent subclinical childhood absence syndrome. Video recordings were not included in the present series, and carbamazepine (CBZ) was employed in only one child who first presented with focal motor seizures and centrotemporal spikes in the EEG. A failed response to CBZ (at potentially toxic serum levels of $52 \mathrm{mg} / \mathrm{L}$ ) was associated after 6 months with a change in the EEG to a synchronous generalized $3 / \mathrm{sec}$ spike and wave pattern, without clinical absence seizures. Replacement of CBZ with valproic acid was followed by clinical rolandic seizure control. All 5 patients responded to valproic acid.

The authors cite 9 previous reports of concomitant EEG findings (frequency usually $7-12 \%$ [25\% and $73 \%$ in two small studies]) but only two reports ( 9 patients) of concomitant clinical absences with focal motor seizures in patients with benign rolandic epilepsy. Perhaps the clinical association of these syndromes might be diagnosed more frequently with simultaneous EEG and video recordings. The concurrence of generalized $3 /$ sec spike and wave discharges with centrotemporal spikes in a child presenting with benign rolandic epilepsy should prompt greater hesitation in starting therapy. When treatment is considered essential, valproic acid would be a better choice than carbamazepine. 